\title{
Promoting Effective Help-Seeking Behavior Through Declarative Instruction
}

\begin{abstract}
The ability to use help-resources efficiently is an important skill in any learning environment, whether the student is in a traditional classroom or is using an Intelligent Tutoring System (ITS). Nevertheless, studies have shown that students' actual help-seeking behavior is far from being ideal (Mandl et al. 2000, Aleven et al. 2001a; 2001b, Ryan et al. 1998).

In this study, we present an evaluation of one way of improving such behavior - by giving the students direct instruction about efficient help-seeking behavior. Two classrooms using the Geometry Cognitive Tutor (Anderson et al. 1995) participated in the study. The instruction to the HELP group, aimed to improve their help-seeking behavior, included a walk-through example. The CONTROL group received instruction with a similar walk-through but without the help-seeking content.

In two subsequent weeks, the HELP group used the system's hints much more frequently than the CONTROL group. Though in general the students showed to learn during the study, we didn't observe a significant difference in the learning outcomes between the two groups.

These results suggest that appropriate instruction can improve help-seeking behavior in ITS usage. Further formative evaluation should be performed in order to design better instruction on help-seeking and to understand its influence on learning outcomes. This approach is only one of several that will be combined in order to try to improve help seeking behavior.
\end{abstract}

\section{Introduction}

One of the most important metacognitive skills in learning is effective help-seeking behavior. Efficient help-seeking behavior in ITS can improve learning outcomes and reduce learning duration (Renkl, 2002; Wood \& Wood, 1999).

The Geometry Cognitive tutor, investigated in this study, is now in extensive use in American public high schools. The tutor uses Bayesian knowledge tracing (Koedinger et al. 1997) to evaluate the knowledge level of students in each skill required for a given question. While some forms of help are given automatically in the tutor (e.g. error messages), ondemand help, which is controlled by the student, can be obtained in two forms:
- Context-sensitive help: At each point during the solution attempt, the student can access multiple levels of hints. The more help the student asks for, the more the question turns into a solved example. Usually the final "bottom-out" hint specifies the required answer to the question. In this unit each question has about three levels of hints.

- Decontextualized help: A Glossary, included as part of the Geometry Cognitive Tutor, includes all the definitions, theorems and formulas that students need. However, being decontextualized, the students must search it and identify the required information in order to make effective use of it.

Aleven et al. (in press) describe a model of desired help seeking behavior in the Geometry Cognitive Tutor. According to that model, a student that has a high chance of solving a question correctly should try to do so, while a student who is very unlikely to solve the question correctly should ask for a hint. Student with an intermediate chance of solving the question correctly should consult the glossary since she has sufficient knowledge to help herself using the glossary as a knowledge base. In addition, after making an error, the student should ask for a hint, in case she does not know how to fix her mistake. After a hint is given, the student should evaluate it before asking for an additional one.

This model should be helpful in leading students to more effective use of help. So far, several studies have shown that students use help in suboptimal ways (Mandl et al. 2000, Aleven et al. 2001a; 2001b). Aleven also found that even when help is used, it is not used in a fashion consistent with the model of desired usage. There is considerable evidence for two types of help misuse:

- Help avoidance, in which students do not use help when they should, especially after making an error or before a first attempt on a new difficult question.

- Help abuse, in which student use help when it is unnecessary, especially when asking for additional hints before evaluating the former ones.

One way to try to improve students' help use is through guiding students to more effective one. White \& Fredriksen (1998) showed that by developing students' metacognitive knowledge and skills the 
students learn better. McNeil \& Alibali (IR) showed that students' goals and strategies can be modified in lab settings by prompting at the beginning of an educational session. These studies suggest that appropriate instruction about desired help seeking behavior might be effective at improving that behavior.

\section{Experiment}

Two classrooms from an urban high school in the Pittsburgh area participated in this study. The study was incorporated into the students' existing curriculum, and the students were proficient in working with the Geometry Cognitive Tutor.

The students were divided into two groups: the HELP group received instruction aimed to improve their help-seeking behavior. The CONTROL group received "placebo-instruction" which focused only on the subject matter without any metacognitive content. The instruction was given through a website, and students read it at their own pace. Both the HELP and CONTROL instruction led the students through solved examples in the unit the students were working on. The HELP instruction incorporated the desired help-seeking behavior into it (see figure 1).

took the one that corresponded to her progress in the curricula. Then the students went individually through the help-seeking instruction, which took about 15 minutes.

On the second day, the students went through additional 5 minutes of instruction. This time they had to solve a question. In addition to the feedback on the cognitive level, which students in both groups received, students in the HELP group received feedback on their help-seeking actions. At the end of the two weeks, the students took a post-test.

\section{Results}

27 students participated in the study (14 of these students were in the HELP group and 13 were in the CONTROL group). Two metrics were used to evaluate the students' work: analysis of logs of the students' usage of the tutor, and a normalized measure of learning gain (which was calculated as the actual improvement from pre- to post-test divided by maximum possible improvement).

As in Wood \& Wood (1999), we calculated the ratio of hints to errors, measured by hints/(hints+errors). This ratio was much higher for the HELP group (0.24) than for the CONTROL group

\begin{tabular}{|l|l|}
\hline $\begin{array}{l}\text { Lets apply the first principle. ask yourself: } \\
\text { Do I have a sense of what to do? }\end{array}$ & Here is what we should do: \\
$\begin{array}{l}\text { To tell you the truth - I don't have a clue about what we should } \\
\text { do. I don't know how to solve it, so I will use the available hints. }\end{array}$ & $\begin{array}{l}\text { First calculate the areas of both a } \\
\text { large pizza and a small pizza. }\end{array}$ \\
\begin{tabular}{|l|l|}
\hline $\begin{array}{l}\text { First calculate the areas of both a large pizza } \\
\text { and a small pizza. }\end{array}$ & $\begin{array}{l}\text { Than compare three small pizzas to a } \\
\text { large one. }\end{array}$ \\
Than compare three small pizzas to a large one. & \\
\hline$<<>$ ok
\end{tabular} \\
\hline
\end{tabular}

Figure 1 - A snapshot from the instruction. Both the HELP instruction (left hand side) and the CONTROL instruction (right hand side) give the same cognitive information. In addition, the help-instruction suggests a metacognitive hint about the right way to obtain that information.

The desired help-seeking behavior, expressed in the instruction, included the following principles:

- Ask for a hint when you don't know what to do, instead of guessing.

- Read the hint before you ask for an additional one,

- Don't guess quickly after committing an error.

The study took place on 4 class days spread out across 2 weeks, during which the students worked on the tutor for approximately three hours. During the first day of the study, students took a pretest. Since the students were in different lessons of the same unit, there were several levels of pretests, and each student
(0.09). This result was marginally significant $(\mathrm{F}(1,21)=2.96, \quad \mathrm{p}=0.10)$, suggesting that the instruction encouraged more frequent help-use. However, this does not answer the question of whether the hint-requests were appropriate.

After the study, the students reported how much attention they paid to the instruction. As might be expected, the students' selfreported attention didn't affect the help-use of the CONTROL group (the hints to errors ratios for both high- and low-attention students were 0.09). However, the students that reported paying low attention in the HELP group used much more help then those reported paying high attention (ratio of 0.12 hints to errors for high-attention students vs. 0.43 for low-attention students). This interaction was significant $(\mathrm{F}(1,11)=8.31, \mathrm{p}=0.01)$. We hypothesize that students who paid low attention to the instruction understood only that they should use help a lot, and thus engaged in hint abuse.

Across the two conditions, the students showed learning during the experiment (out of 4 possible points in each test, the average pre-test score was 1.15 and the post-test score was 1.67. This improvement was significant, $\mathrm{T}(0,26)=2.10, \mathrm{p}=0.04)$. 
Direct comparison between conditions was difficult, given the design of our study where students were working on different tutor lessons, and thus we did not observe any significant influence of the condition on the learning outcomes.

\section{Conclusions and Future Work}

In this study we show that help-seeking instruction has the potential to influence help-seeking behavior in real classroom environments. However, more studies should be done to determine how students' actual help-seeking behavior changes after such instruction, and what the impact of the instruction is (i.e., how does it influence learning outcomes, for how long the influence persists and whether it extends to other tutor lessons).

Most important, the help-seeking instruction should be combined with supporting tools such as tracing the students' help seeking behavior in real time, supplying tailored feedback, and promoting selfassessment and self-explanation.

\section{Acknowledgements}

We would like to thank Matthew Welch and Michele O'Farrell for assisting us in conducting this study, and Ryan S. Baker for helpful suggestions and comments.

\section{Reference}

- Aleven, V., \& Koedinger, K. R. (2000). Limitations of student control: Do students know when they need help? In C. F. G. Gauthier \& K. VanLehn (Eds.), Proceedings of the 5th International Conference on Intelligent Tutoring Systems, ITS 2000 (pp. 292-303).Berlin: Springer Verlag.

- Aleven, V. (2001, October). Helping students to become better help seekers: Towards supporting metacognition in a Cognitive Tutor. Paper presented at the 1st NSF-DFG Workshop on Learning Technologies, Tübingen, Germany. (unpublished).

- Aleven V. \& Koedinger, K. R. (2001). Investigations into help seeking and learning with a Cognitive Tutor. In R. Luckin (Ed.), Papers of the AIED-2001 Workshop on Help Provision and Help Seeking in Interactive Learning
Environment s ( p p . $47-58)$ ). http://www.hcrc.ed.ac.uk/aied2001/workshops.html

- Aleven, V., Stahl, E., Schworm, S., Fischer, F., \& Wallace, R.M. (2003). Help Seeking and Help Design in Interactive Learning Environments. Review of Educational Research, 73(2), 277-320.

- Aleven, V., McLaren, B., Roll, I. \& Koedinger, K. R. (in press). Toward tutoring help seeking - applying cognitive modeling to meta-cognitive skills. To appear at ITS 2004.

- Anderson, J. R., A. T. Corbett, K. R. Koedinger, and R. Pelletier, (1995). Cognitive tutors: Lessons learned. The Journal of the Learning Sciences, 4, 167-207.

- Clark, R. C. \& Mayer, R. E. (2003), e-Learning and the Science of Instruction. Jossey-Bass/Preiffer, San Francisco, USA.

- Koedinger, K.R., Anderson, J.R., Hadley, W.H., \& Mark, M.A. Intelligent Tutoring Goes to School in the Big City. (1997) International Journal of Artificial Intelligence in Education, 8, 30-43.

- Mandl, H., Gräsel, C. \& Fischer, F. (2000). Problemoriented learning: Facilitating the use of domain-specific and control strategies through modeling by an expert. In W. J. Perrig \& A. Grob (Eds.), Control of Human Behavior, Mental Processes and Consciousness (pp.165-182). Mahwah: Erlbaum.

- McNeil, N.M. \& Alibali, M.W. (2000), Learning Mathematics from Procedural Instruction: Externally Imposed Goals Influence What Is Learned, Journal of Educational Psychology, 92 \#4, 734-744.

- Renkl, A. (2002). Learning from worked-out examples: Instructional explanations supplement self-explanations. Learning \& Instruction, 12, 529-556.

- Ryan, A. M., Gheen, M. H. \& Midgley, C. (1998), Why do Some Students Avoid Asking for Help? An Examination of the Interplay Among Students' Academic Efficacy, Teachers' Social-Emotional Roke, and the Classroom Goal Structure. Journal of Educational Psychology, 90 (3), 528535.

- White, B.Y. \& Federistan, J.R. (1998), Inquiry, Modeling, and Metacognition: Making Science Accessible to All Students. Cognition and Instruction, 16(1), 3-118

- Wood, H., \& Wood, D. (1999). Help seeking, learning and contingent tutoring. Computers and Education, 33, 153169. 